\title{
Crystallization Engineering in Aza-Steroid: Application in the Development of Finasteride
}

Apurba Bhattacharya ${ }^{\mathrm{b}}$, Srinivasula Reddy Maddula $^{\mathrm{a}}$, B. R. Sreekanth ${ }^{\mathrm{c}}$, Kushal S. Manudhane ${ }^{\mathrm{a}}$, Sridhar Thota ${ }^{\mathrm{a}}$, Rakeshwar Bandichhor ${ }^{\mathrm{a}^{*}}$

${ }^{a}$ Center of Excellence, Research \& Development, Integrated Product Development, Dr. Reddy's Laboratories Ltd., Survey Nos. 42,45,46, \& 54 Bachupally, Qutubullapur, Ranga Reddy Dist 500072, Andhra Pradesh, India

${ }^{b}$ Department of Chemistry, Texas A\&M University, Kingsville, TX 78363, USA

${ }^{c}$ Department of Analytical Research, Discovery Research, Dr. Reddy's Laboratories Ltd., Miyapur, Hyderabad 500049, Andhra Pradesh, Indid *Corresponding author. Tel.: +918458279 485; Fax: +91 8458279619.

Email: rakeshwarb@drreddys.com

\section{Supporting Information}

1. Purification details

Page S2

2. Details of recovery of the purified material that was used to pack the column.

Page S3

3. Analytical techniques experimental details

Page S3

4. ${ }^{1} \mathrm{H}-\mathrm{NMR}$ correlation studies

Page 84

5. Solid state ${ }^{13} \mathrm{C}$-NMR correlation studies

6. IR correlation studies

Page S5

Page S6

7. Powder X-Ray Diffraction (PXRD) comparison

Pages S6-S7

8. Differential Scanning Calorimetry (DSC) comparison

Page S8

9. Thermo Gravimetric Analysis (TGA) comparison

Page S9 


\section{Purification details}

Complete oxidation of intermediate $\mathbf{2}$ to afford $\mathbf{1}$ has not been practically realized. The un-reacted 2 contributes at least 0.5 - $3 \%$ impurity with excellent yield of 1 . A further attempt to oxidize un-reacted $\mathbf{2}$ appears to be impractical since this leads to over oxidation. The isolation of impurity $\mathbf{2}$ as un-reacted intermediate, in the DDQ and BSTFA oxidation step towards the synthesis of $\mathbf{1}$, was accomplished by utilizing solid solution formation technique. Such a concept although seemingly implausible was surmised by experiment employing simple filteration of a solution of $\mathbf{1}$ (containing 1-3\% of 2) through a short column of $\mathbf{1}$ (pad filtration) where $\mathbf{1}$ essentially acts as a molecular trap and binds the $\mathbf{2}$ molecules in an irreverstible manner; the eluent was essentially free of the impurity $2(<0.2 \%)$ providing deliverable quality of the drug substance.

A slight modification provides same results (Table 1). Instead of using glass column, a saturated solution of impure finasteride (97\% pure) was added to a pure finasteride 1 in a round bottom flask equipped with mechanical stirrer. After stirring for 1 h, solid material was filtered. HPLC chromatogram of mother liquor shows $99.8 \%$ purity that reflects successful solid solution formation of dihyrdo-impurity on residual finasteride solid. The mother liquor was concentrated to obtain finasteride 1 with $0.2 \%$ impurity of 2 in $97 \%$ yield HPLC analysis of residual solid confirms the uptake of impurity 2. HPLC quantification, of impurity $\mathbf{2}$ in finasteride $\mathbf{1}$, that was estimated via continuous as well as batch mode protocols are summarized in Table 1.

Table 1. HPLC quantification of impurity 2.

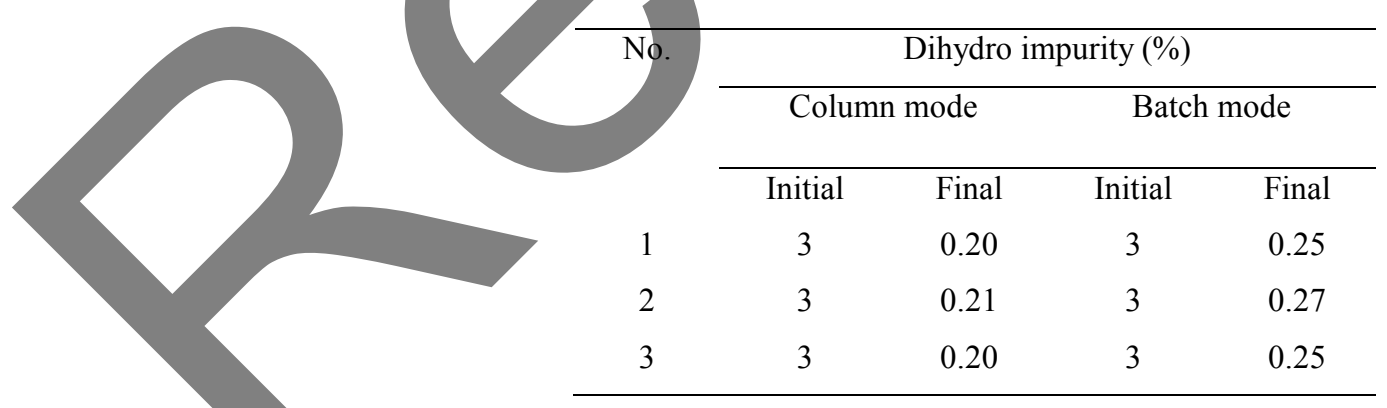




\section{Details of recovery of the purified material that was used to pack the column.}

\section{a) Experiment 1.}

$10 \mathrm{~g}$ of finasteride containing 3\% DHF impurity was dissolved in $800 \mathrm{~mL}$ of ethyl acetate to prepare saturated solution. This saturated solution obtained above was passed, at a rate of $0.4 \mathrm{~mL} / \mathrm{min}$., through the $15 \mathrm{gm}$ of pure finasteride powder (solid) packed in $1.1 \mathrm{~cm} \mathrm{X} 10 \mathrm{~cm}$ glass column with 1-2 cm glass beads of 90-150 $\mu$ packed from both the end of column.

After completion of elution, either in continuous or batch mode, the impurity level of finastaride 1 drops down to 0.20 to $0.25 \%$ from initial $3 \%$. After evaporating the solvent dry powder obtained in $97 \%$ yield $(\sim 9.7 \mathrm{~g})$ and the yield of the solid powder recovered from column (continuous mode) or flask (batch mode) was about $102 \%(\sim 15.28 \mathrm{~g})$.

\section{b) Experiment 2.}

$15 \mathrm{~g}$ of finasteride containing 3\% DHF impurity was dissolved in $1200 \mathrm{~mL}$ of ethylacetate to prepare saturated solution. This saturated solution obtained above was passed, at a rate of $0.4 \mathrm{~mL} / \mathrm{min}$., through the $10 \mathrm{gm}$ of pure finasteride powder (solid) packed in $1.1 \mathrm{~cm} \mathrm{X}$ $10 \mathrm{~cm}$ glass column with 1-2 cm glass beads of 90-150 $\mu$ packed from both the end of column.

After completion of elution, either in continuous or batch mode, the impurity level of finastaride 1 drops down to 0.20 to $0.25 \%$ from initial $3 \%$. After evaporating the solvent dry powder was obtained in $\sim 95 \%$ yield $(\sim 14.2 \mathrm{~g})$.

\section{Analytical techniques experimental details}

Polymorphic form-HI of finasteride $\mathbf{1}$ was used in all the experiments. Solvents and regents were used for all the reactions as received. Solid state ${ }^{13} \mathrm{C}$-NMR was recorded at $300 \mathrm{MHz}$. Infrared (IR) spectra were recorded as thin films on a Mattson Galaxy Series FTIR 3000 spectrometer referenced to polystyrene standard. X-ray powder diffraction has been collected on the Rigaku D/Max-2200 model diffractometer equipped with horizontal goniometer in $\theta / 2 \theta$ geometry. $\mathrm{Cu}-\mathrm{K} \alpha(\lambda=1.5418 \AA)$ radiation was used and the samples were scanned between $3-45^{\circ} 2 \theta$. Differential Scaning Calorimetric (DSC) analyses were carried out on Shimadzu DSC50. The ThermoGravimetric Analysis (TGA) was performed on Q500 of TA Instruments. The thermogram was recorded from 25 to $250{ }^{\circ} \mathrm{C}$ under the nitrogen gas purge at a flow of $40 \mathrm{~mL} / \mathrm{min}$ for balance and 60 $\mathrm{mL} / \mathrm{min}$ for sample at a heating rate of $10^{\circ} \mathrm{C} / \mathrm{min}$. All the experiments were conducted with the solid (either finasteride and dihydrofinasteride are in 1:1 or 1:2 ratio as input) 
material obtained from the solution in appropriate solvents like EtOAc or EtOH either by distillation or slow evaporation.

\section{4. ${ }^{1}$ H-NMR correlation studies}

\section{${ }^{1} \mathrm{H}$ NMR Correlation}

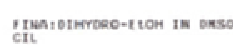

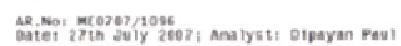

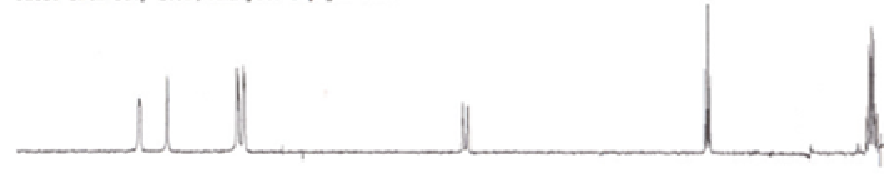

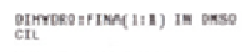

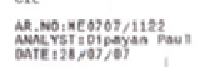

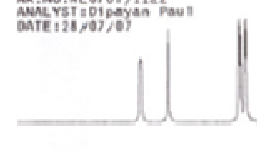

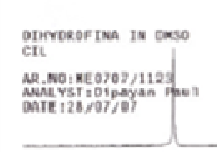

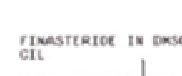
and

Figure: ${ }^{1} \mathrm{H}-\mathrm{NMR}$ of (a) Finasteride (b) Dihydrofinasteride (c) 1:1 physical mixture (d) Single crystal of solid solution (which was mounted to record the X-ray). 


$$
e^{e^{x^{\alpha^{2}}}}
$$


6. IR correlation studies (Finasteride and Dihydrofinasteride have been prepared separately to generate control samples): The indicated value in each panel corresponds to amide $-\mathrm{C}=\mathrm{O}$.
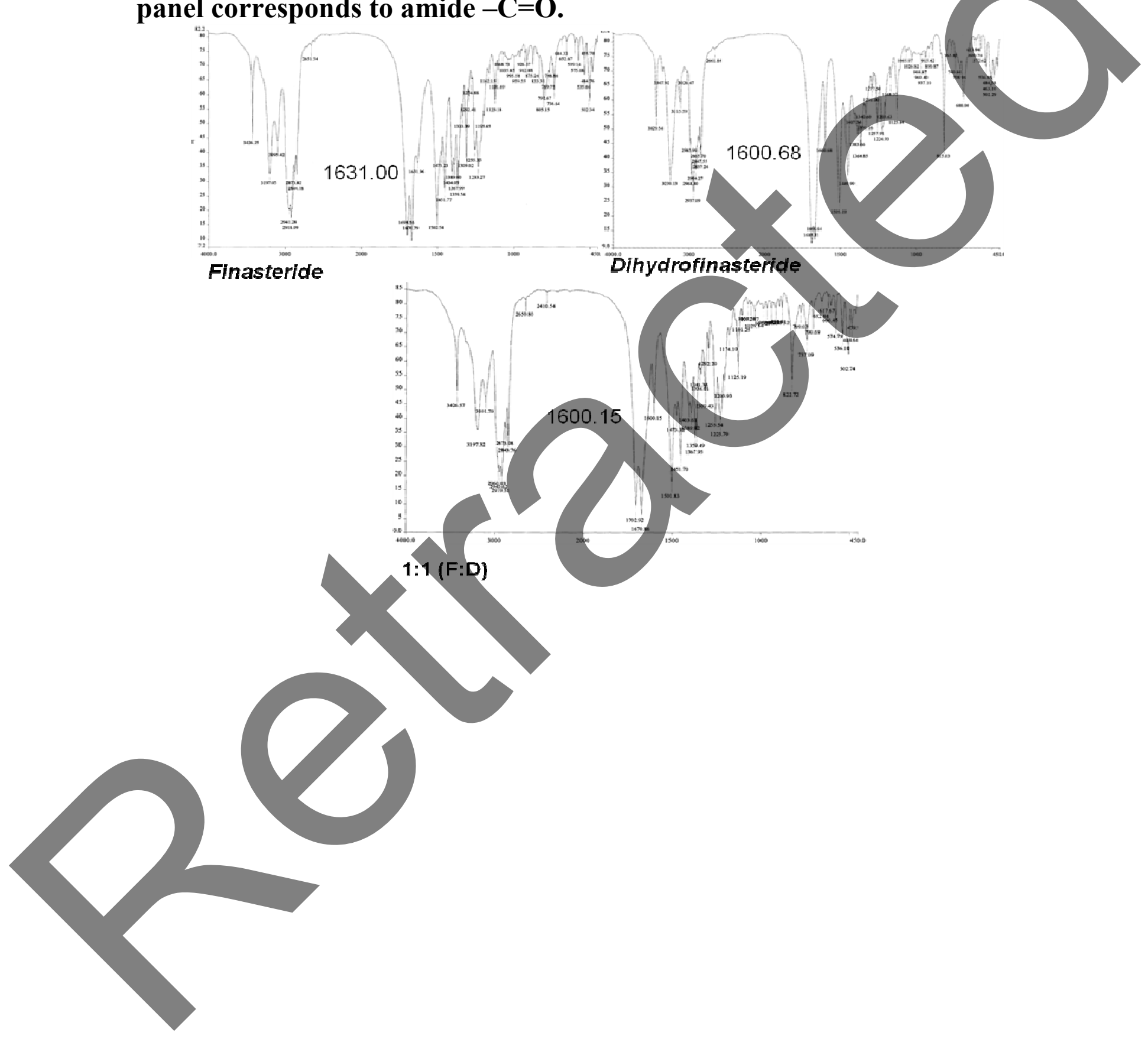
7. Powder X-Ray Diffraction (PXRD) comparison (Finasteride and Dihydrofinasteride have been prepared separately to generate control samples)
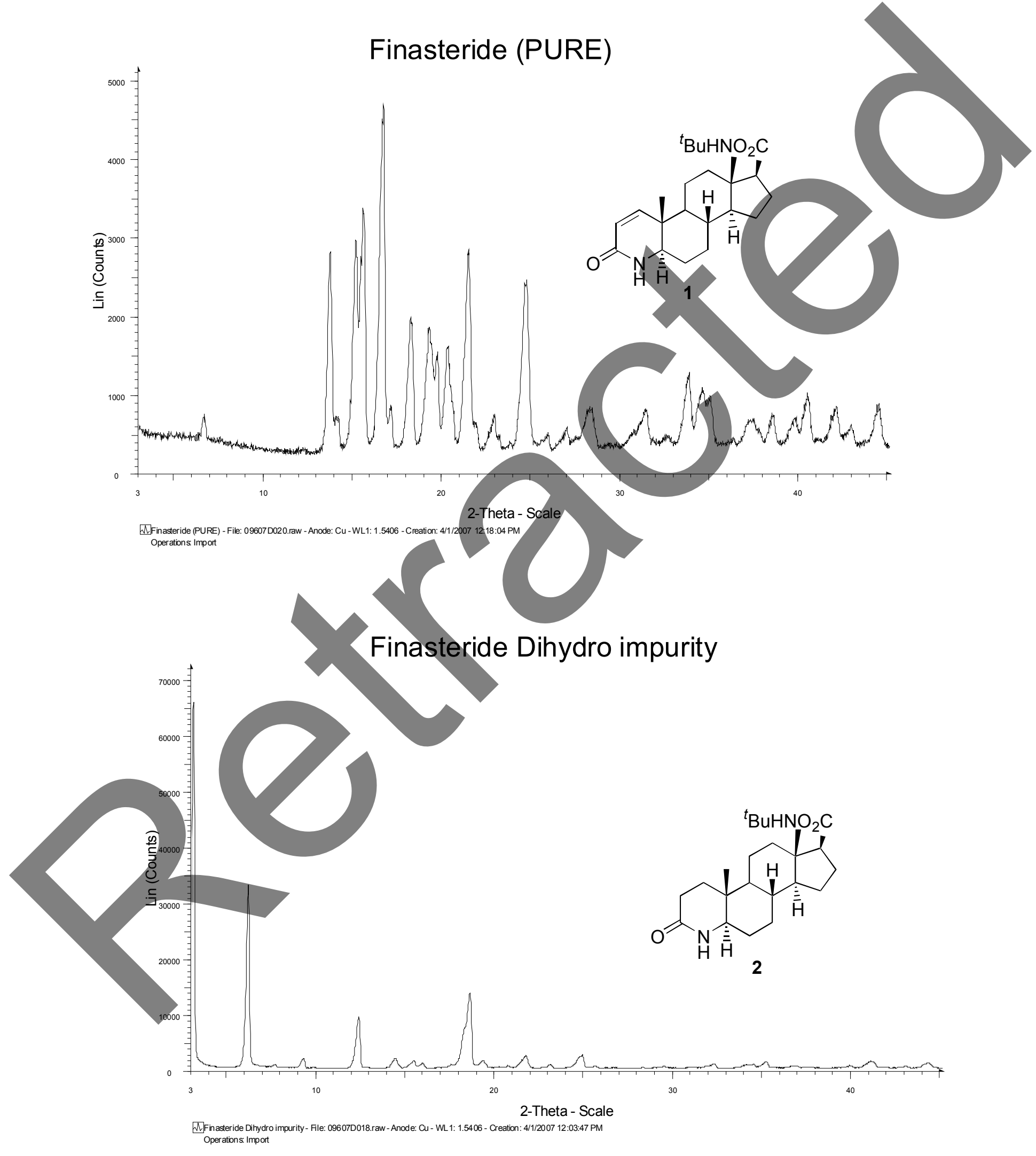


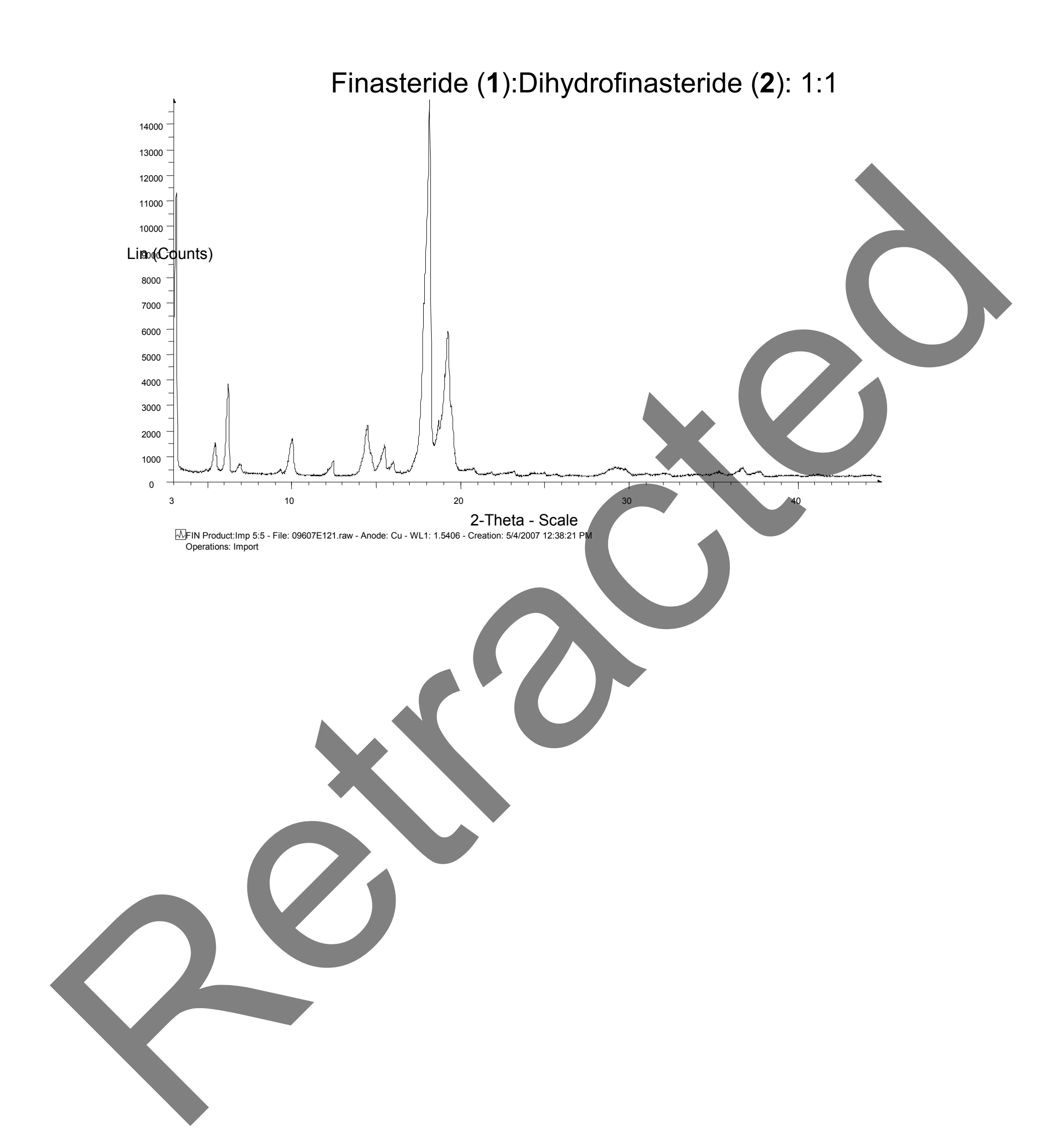


<smiles>C1=C[C@@H]2C[C@@H]12</smiles> 
<smiles>C1=C[C@@H]2C[C@@H]12</smiles> 